\title{
Estudios Adsorción de Ácido Ferúlico en Quitosán
}

\author{
H.J. Amézquita García ， F.J. Cerino Córdovaa , M.N. Sánchez-González ', E. Soto Regalado, N. E. Dávila \\ Guzman $^{a}$, J.A. Loredo Medrano
}

${ }^{a}$ Universidad Autónoma de Nuevo León, Facultad de Ciencias Química, Av Universidad, Cd. Universitaria, San Nicolás de los Garza, N.L., 66451,México.

*E-mail:felipejccuanl@yahoo.com.mx

Recibido 16 de mayo de 2012, Aceptado 5 de junio de 2012

\section{Resumen}

En este artículo se evalúa la factibilidad de utilizar el quitosan como adsorbente para la recuperación de ácido ferúlico de soluciones acuosas. El efecto del pH sobre la capacidad de adsorción fue estudiada, siendo a pH 2 en donde se obtiene la mejor capacidad de adsorción (80 mg $\mathrm{AF} / \mathrm{g}$ ), además se observo una disminución de la capacidad del quitosan para adsorber ácido ferúlico conforme se incrementa el pH. En los estudios cinéticos, se demostró que el tiempo para alcanzar el equilibrio fue de $60 \mathrm{~min}$ independientemente de la concentración de adsorbente utilizado y el modelo de pseudo- primer orden fue el modelo que mejor se ajusto a los datos experimentales. Estudios de equilibrio de adsorción fueron realizados a diferentes temperaturas $\left(30-50{ }^{\circ} \mathrm{C}\right)$, la capacidad de adsorción disminuyo conforme se incrementa la

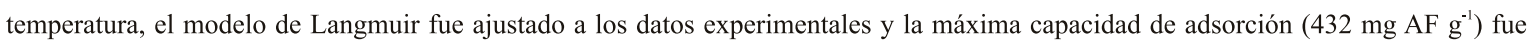
obtenida a $30^{\circ} \mathrm{C}$.

Palabras clave: Adsorción, Quitosán, Ácido Ferúlico, Compuesto fenólico

\begin{abstract}
In this paper, the feasibility to use chitosan as a low-cost adsorbent of ferulic acid from aqueous solution was evaluated. The effect of $\mathrm{pH}$ solution on adsorption capacity was studied; the best capacity adsorption $(80 \mathrm{mg} \mathrm{AF} / \mathrm{g})$ was obtained at $\mathrm{pH} 2$. A decrease of the capacity of chitosan to adsorb ferulic acid with increasing $\mathrm{pH}$ was observed. Kinetics experiments showed that the adsorption equilibrium was achieved in $60 \mathrm{~min}$; it was independently of adsorbent dose. The pseudo-first order model fitted well to kinetic experimental data. Adsorption equilibrium studies were carried out in temperature range from $3^{\circ}$ to $50^{\circ} \mathrm{C}$, the adsorption capacity decreased with increasing temperature,
\end{abstract}

\section{Introducción}

El ácido ferúlico (AF) es un compuesto de alto valor agregado que ha sido extensamente estudiado, actualmente las investigaciones se orientan hacia el diseño de tratamientos para su extracción de fuentes naturales, así como a la biotransformación del mismo para la obtención de productos de alto valor agregado [1]. En Estados Unidos y en la gran mayoría de países europeos, numerosos extractos naturales y esencias medicinales de hierbas, café, vainilla, especias y otras sustancias de origen botánico, son seleccionados por su alto contenido en AF para ser añadidos a los alimentos como antioxidantes (aprobados por la FDA) [2]. En México, la industria de la tortilla genera residuos sólidos y líquidos que pudieran revalorizare para la obtención de moléculas valiosas como es el caso del AF.

En la parte de los residuos sólidos generado, el cual tiene un alto contenido de AF, se tiene el pericarpio de maiz, pero es necesario realizar su hidrólisis para obtener el AF en soluciones acuosas [3]. Por lo tanto, se requiere un método para recuperar el $\mathrm{AF}$ de soluciones acuosas, sien el método de adsorción una vía factible de explorar, por lo cual se requiere seleccionar un adsorbente altamente afín a este compuesto. La elección del mejor adsorbente, se basa en el impacto que tienen diversos factores como el $\mathrm{pH}$, el tiempo de adsorción al equilibrio y la temperatura, sobre la capacidad de adsorción. En el presente trabajo se realizó un análisis cinético y termodinámico de la adsorción del AF sobre Quitosán (QUI) en solución acuosa.

\section{Parte experimental}

\subsection{Tratamiento del adsorbente}

Las propiedades del adsorbente quitosán utilizado en este estudio son mostradas en la tabla 1. El quitosán fue sometido a varios ciclos de lavado con agua destilada hasta obtener un $\mathrm{pH}$ constante en el agua de lavado. Posteriormente, el quitosán se seco a $45^{\circ} \mathrm{C}$ durante 3 días y almacenado herméticamente a temperatura ambiente hasta su uso en las pruebas de adsorción.

Tabla 1. Propiedades del quitosan.

\begin{tabular}{ll}
\hline Forma física & Polvo blanco-amarillo. \\
Capacidad de retención de Humedad & $79 \%$ (Valor promedio) \\
Grado de desacetilación & $>85 \%$ \\
Tamaño de partículas & 80 mallas $\approx 177 \mu \mathrm{m}$ \\
\hline
\end{tabular}

\# Dato calculado.

\subsection{Influencia del $\mathrm{pH}$ sobre la capacidad de adsorción de $\mathrm{AF}$ en quitosan}

Los experimentos de adsorción tipo Batch a $40^{\circ} \mathrm{C}$, fueron llevados a cabo por triplicado agregando $0.14 \mathrm{~g}$ de QUI en base seca, a matraces Erlenmeyer de 250 $\mathrm{mL}$ conteniendo $25 \mathrm{~mL}$ de una solución de $1 \mathrm{~g} \mathrm{AF} \mathrm{L}^{-1}$ a diferentes valores de $\mathrm{pH}(2-6)$. El pH de la solución inicial de AF se ajustó con la adición de una solución de $6 \mathrm{~N} \mathrm{HC}^{l}$ ó $2.4 \mathrm{M} \mathrm{NaOH}$. Los matraces fueron sometidos a agitación a una velocidad de $125 \mathrm{rpm}$. Muestras fueron extraidas al inicio y final de la etapa experimental (200 min). El AF en solución fue analizado por la técnica del Folin-Ciocalteu. 


\subsection{Experimentos de cineticas e isotermas de adsorción}

Los experimentos de cinéticas de adsorción fueron llevados a cabo por triplicado en un sistema tipo lote. Diferentes cantidades conocidas de adsorbente $(0.125$ y $0.625 \mathrm{~g}$ de adsorbente) fueron añadidas a matraces Erlenmeyer de $250 \mathrm{ml}$ conteniendo $25 \mathrm{ml}$ de solución de $\mathrm{AF}\left(1 \mathrm{~g} \mathrm{~L}^{-1}\right)$ a pH 3. Los matraces fueron agitados a una velocidad de $125 \mathrm{rpm}$ y $30^{\circ} \mathrm{C}$. Muestras fueron extraídas del sistema en un intervalo de tiempo de 0 a $200 \mathrm{~min}$

En el caso de los experimentos de equilibrio de adsorción, estos fueron realizados a diferentes temperaturas $\left(30-50^{\circ} \mathrm{C}\right)$, añadiendo diferentes cantidades conocidas de adsorbente $(0.075 \mathrm{a} 0.625 \mathrm{~g}$ de adsorbente en base húmeda) a matraces Erlenmeyer de $250 \mathrm{~mL}$ conteniendo $25 \mathrm{~mL}$ de solución de AF. Las muestras obtenidas en los experimentos de cinética e isotermas de adsorción fueron evaluadas por el método de Folin Ciocalteau.

\subsection{Modelos cinéticos}

El modelo de Pseudo-primer orden (PPO) y Pseudo segundo orden (PSO) fueron utilizados para la modelación de las cineticas de adsorción y se representan por las Ecs. 1 y 2 , respectivamente.

$$
\begin{aligned}
& \log \left(q_{e q}-q\right)=\log q_{e q}-\frac{k_{1} * t}{2.303} \\
& \frac{t}{q}=\frac{1}{k_{2} * q_{e q}^{2}}+\frac{t}{q_{e q}}
\end{aligned}
$$

Donde $\mathrm{q}$ es la cantidad de AF adsorbida al tiempo $\mathrm{t}$ ( $\left.\mathrm{mg} \mathrm{g}^{-1}\right)$; qeq es la concentración de la fase sólida al equilibrio, $\mathrm{t}$ es el tiempo (min) y k1 y k2 son las constantes de adsorción de pseudo-primer orden (min1) y pseudo-segundo orden $\left(\mathrm{g} \mathrm{mg}^{-1} \mathrm{~min}^{-1}\right)$.

Los parámetros de los diversos modelos cinéticos se determinaron con la ayuda de la herramienta de Solver ${ }^{\circledR}$ Microsoft Excel minimizando la función error mostrada en la Ec. 3:

$$
\operatorname{APE}=\frac{\mathbf{1}}{\mathrm{n}} \sum_{\mathrm{i}=1}^{\mathrm{n}}\left|\frac{\mathrm{q}_{\text {exp }, \mathrm{i}}-\mathrm{q}_{\text {calc }, \mathrm{i}}}{\mathbf{q}_{\text {exp, } \mathrm{i}}}\right|
$$

\section{Discusión de resultados}

3.1 Efecto del pH inicial de la solución sobre la capacidad de adsorción

En la Figura 1, se muestra el efecto del $\mathrm{pH}$ de la solución de AF sobre la capacidad de adsorción del quitosan. La capacidad de adsorción de $\mathrm{AF}$ disminuye conforme se incrementa el valor de $\mathrm{pH}$, la mejor capacidad de adsorción de AF sobre QUI (81.9 mg g1), fue obtenida a $\mathrm{pH} 2$ y llega a ser nulas a valores de pH 5 y 6 . Este comportamiento es atribuido a la disociación del AF, en el diagrama de especiación [4] se obtuvo que a los valores de $\mathrm{pH} 5$ y 6 el AF se encuentra totalmente disociado. A valores de $\mathrm{pH}$ inferiores a 5, el grupo funcional amino del QUI tiende a protonarse [5], lo cual resulta en interacciones iondipolo más fuerte del QUI con el AF en su forma no disociada, obteniéndose capacidades de adsorción mayores a valores de $\mathrm{pH}$ ácidos. En los estudios subsecuentes se decidió utilizar $\mathrm{pH} 3$ para efectuar los estudios cinéticos y las isotermas de adsorción, dado que las soluciones de $1 \mathrm{gAF} \mathrm{L}^{-1}$ preparadas presentaron un $\mathrm{pH} 3.2$.

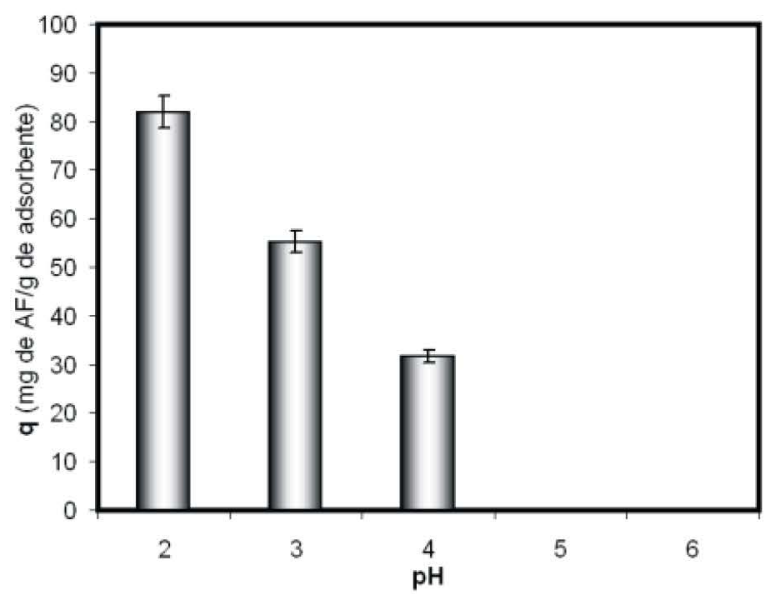

Figura 1. Efecto del pH inicial de la solución de AF.

\subsection{Estudio cinético}

La información de las cinéticas de adsorción en la recuperación del $\mathrm{AF}$ es requerida para la selección de las condiciones óptimas de operación de un proceso de adsorción a gran escala. En la figura 2, se muestran los datos cinéticos obtenidos para la adsorción de $\mathrm{AF}$ sobre QUI A una temperatura de $40^{\circ} \mathrm{C}$ y concentración de adsorbente de $5 \mathrm{~g} \mathrm{~L}^{-1}$. El tiempo de equilibrio de adsorción a $40^{\circ} \mathrm{C}$ es aproximadamente 60 minutos, e independiente de la concentración de adsorbente ( 5 o $25 \mathrm{~g} \mathrm{AF} \mathrm{L}^{-1}$ ). Los datos cinéticos obtenidos, fueron ajustados mediante la ecuación de pseudo-primer orden (PPO) y pseudo-segundo orden (PSO). Los resultados de la tabla 2 indican que el modelo de PPO se ajusta mejor a los datos experimentales para el QUI, con un coeficiente de correlación de $r_{2}=0.971$, y un porcentaje promedio de error de $\% \mathrm{APE}=6.58$. 
Tabla 2. Parámetros de los modelos cinéticos y porcentajes de error de las cinéticas de adsorción.

\begin{tabular}{|c|c|c|c|c|c|c|c|}
\hline \multirow{2}{*}{$\begin{array}{c}\text { Concentración } \\
\text { Adsorbente } \\
\left(\mathrm{g} \mathrm{L}^{3}\right)\end{array}$} & \multirow[t]{2}{*}{ Modelos Cinéticos } & \multicolumn{6}{|c|}{ Parámetros } \\
\hline & & & & $\begin{array}{l}\text { qugies }^{-1} \\
\left(\mathrm{mg}^{-1}\right)\end{array}$ & $\left(\begin{array}{c}q_{\text {quepp }} \\
\left(\mathrm{mgg}^{-3}\right)\end{array}\right.$ & $\boldsymbol{R}^{2}$ & $\stackrel{\%}{A P E}$ \\
\hline \multirow{2}{*}{$5 \mathrm{~g} / \mathrm{L}$} & PPO & $\mathbf{k}_{\mathbf{1}}=$ & 0.026 & 318 & 318 & 0.971 & 6.58 \\
\hline & PSO & $\mathbf{k}_{2}-$ & $1 \times 10^{4}$ & 333 & & 0.956 & 8.20 \\
\hline \multirow{2}{*}{$25 \mathrm{~g} / \mathrm{L}$} & PPO & $\mathbf{k}_{1}=$ & 0.058 & 184 & 187 & 0.897 & 5.26 \\
\hline & PSO & $k_{2}-$ & $6 \times 10^{-4}$ & 199 & 187 & 0.884 & 4.95 \\
\hline
\end{tabular}

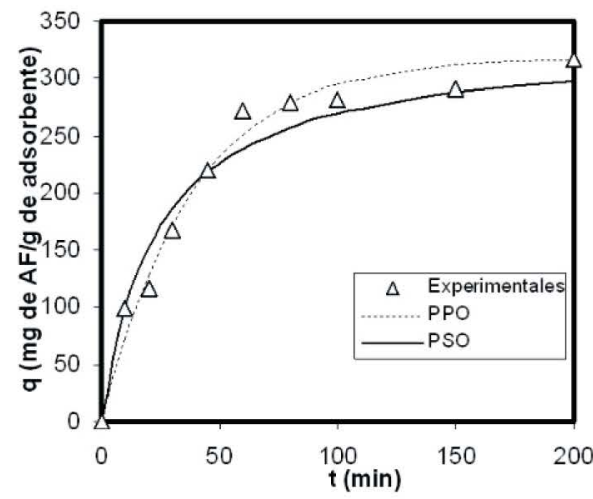

Figura 2. Cinetica de adsorción de AF en Quitosán a concentración de adsorbente de 5 g L-1.

\subsection{Estudio de equilibrio}

En la figura 3, se muestran las isotermas de adsorción de $\mathrm{AF}$ sobre QUI obtenidas en un rango de temperatura de 30 a $50^{\circ} \mathrm{C}$. La capacidad de adsorción tiende a disminuir con el incremento de temperatura. La mejor capacidad de adsorción se obtuvo a una temperatura de $30^{\circ} \mathrm{C}$, para aplicaciones a gran escala este es un resultado positivo dado que no se requeriría agregar energía al proceso de adsorción. Del análisis termodinámico se obtuvo que el proceso es espontaneo y endotérmico. Los datos de equilibrio de adsorción de $\mathrm{AF}$ en quitosán fueron ajustados mediante los modelos de Langmuir (LA), Freundlich (FR) y Redlich-Peterson (RP) mostrados en la tabla 3.

Los parámetros calculados de las isotermas de adsorción de AF en QUI en el intervalo de $30-50^{\circ} \mathrm{C}$ son mostrados en la tabla 4. Los modelos mejor ajustados (mayores valores de $\mathrm{R}_{2}$ y menores valores de $\% \mathrm{APE}$ ) se clasificaron de la siguiente manera: LA > RP > FR. Los altos valores de $\mathrm{R}_{2}>0.9607$ y el valor medio global del \%APE de 4.4\%, mostraron que el mejor ajuste a los datos experimentales se obtiene usando el modelo de LA. De acuerdo a la isoterma de LA, el AF es adsorbido en la superficie del adsorbente QUI en forma de monocapa ideal. Los valores de capacidad máxima de adsorción ajustados en el modelo de LA, son ligeramente mayores al rango de valores que toma el parámetro $\mathrm{q}_{\exp }(306-376 \mathrm{mg} \mathrm{de}$ $\mathrm{AF} / \mathrm{g}$ de QUI).
Tabla 3. Modelos de isoterma de adsorción utilizados.

\begin{tabular}{|c|c|c|}
\hline $\begin{array}{l}\text { Modclo } \\
\text { Isoterma }\end{array}$ & Modelo & $\begin{array}{ll}\text { Paranetros } \\
\end{array}$ \\
\hline Langmuir & $q=\frac{q_{\max } b C_{\mathrm{eq}}}{1+b C_{\mathrm{eq}}}$ & $\begin{array}{l}\mathrm{q}=\text { adsorption capacity }\left(\mathrm{mg} \mathrm{g}^{-1}\right) \\
\mathrm{C}_{\mathrm{eq}}=\text { Concentración al equilibrio en la fase liquida (mg } \mathrm{L}^{-1} \text { ) } \\
\left.\text { qmax = Capacidad máxima de adsorción (mg g } \mathrm{g}^{-1}\right) \\
\mathrm{b}=\text { Constante Langmuir }\left(\mathrm{L} \mathrm{mg}^{-1}\right)\end{array}$ \\
\hline Froundlich & $q=K_{F} C_{c y}^{1 / 0}$ & $\mathrm{~K}_{\mathrm{F}}=$ Constante de Fremdlich (mg $\left.{ }^{1-1 / n} \mathbf{g}^{1 / /} \mathbf{L}^{1 / n}\right)$ \\
\hline $\begin{array}{l}\text { Redichl- } \\
\text { Peturson }\end{array}$ & $q=\frac{K_{p p q} C_{\mathrm{eq}}}{1+A_{\mathrm{Rp}} C_{\mathrm{gq}}^{m}}$ & $\begin{array}{l}K_{\mathrm{kP}}=\text { Constante de Redlich-Peterson }\left(\mathrm{L}^{-1}\right) \\
\mathrm{A}_{\mathrm{kP}}-\text { Constante de Redich-Peterson }\left(\mathrm{L} \mathrm{mb}^{1}\right)^{\mathrm{m}} \\
\mathbf{m}=\text { Constante de Redlich-Peterson }\end{array}$ \\
\hline
\end{tabular}

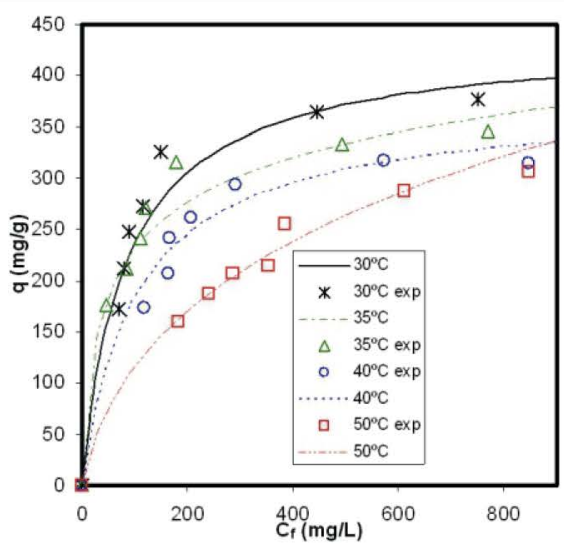

Figura 3. Isotermas de Adsorción de ácido ferúlico en quitosan.

Tabla 4. Parámetros de las isotermas para el QUI a diferentes temperaturas

\begin{tabular}{|c|c|c|c|c|c|c|}
\hline \multirow[t]{2}{*}{$\begin{array}{l}\text { Moddo } \\
\text { isotérmico }\end{array}$} & \multirow[t]{2}{*}{$\mathbf{T}(\mathbf{K})$} & \multicolumn{5}{|c|}{ Parámetros del modelo } \\
\hline & & $9 \max$ & b & & $\mathbf{R}^{2}$ & $\%$ APE \\
\hline \multirow[b]{4}{*}{ Langmuir } & 303 & 432 & 0.0120 & & 0.9651 & 6.2 \\
\hline & 308 & 366 & 0.0207 & & 0.9835 & 3.5 \\
\hline & 313 & 400 & 0.0065 & & 0.9607 & 5.6 \\
\hline & 323 & 415 & 0.0034 & & 0.9912 & 2.2 \\
\hline \multirow[b]{5}{*}{ Freundlich } & & $\mathbf{K}_{\mathbf{F}}$ & n & & & \\
\hline & 303 & 70.02 & 3.69 & & 0.9267 & 9.5 \\
\hline & 308 & 75.62 & 4.18 & & 0.9485 & 6.3 \\
\hline & 313 & 50.14 & 3.44 & & 0.9401 & 8.1 \\
\hline & 323 & 15.51 & 2.20 & & 0.9808 & 3.4 \\
\hline \multirow{5}{*}{$\begin{array}{l}\text { Redilich } \\
\text { Peterson }\end{array}$} & & $K_{\text {KRP }}$ & $\mathbf{A}_{\mathbf{K R}}$ & $\mathbf{m}$ & & \\
\hline & 303 & 5.3 & 0.013 & 0.9921 & 0.9642 & 6.3 \\
\hline & 308 & 13.1 & 0.089 & 0.8610 & 0.9686 & 4.3 \\
\hline & 313 & 3.7 & 0.010 & 0.9966 & 0.9733 & 5.9 \\
\hline & 323 & 2.8 & 0.057 & 0.6940 & 0.9868 & 3.0 \\
\hline
\end{tabular}

\section{Conclusiones}

En el presente estudio se ha demostrado la factibilidad de utilizar el quitosán para la adsorción de ácido ferúlico. $\mathrm{El} \mathrm{pH}$ tiene una gran influencia en la adsorción de ácido ferúlico sobre quitosán, obteniéndose la máxima capacidad de adsorción a $\mathrm{pH}$ 2. Al incrementar el valor de $\mathrm{pH}$ de la solución a 5 y 6 , se obtuvo una capacidad de adsorción nula, a causa de la baja afinidad que existe entre el ion ferulato y el quitosán. Los datos experimentales obtenidos en las isotermas para el Quitosán, se ajustaron mejor al modelo de Langmuir, obteniéndose una capacidad máxima de adsorción a $30^{\circ} \mathrm{C}$ de $432 \mathrm{mg} \mathrm{AF} \mathrm{g}^{-1}$. Los estudios demuestran la factibilidad de utilizar el quitosan como un adsorbente de bajo costo para la adsorción de ácido ferúlico proveniente de la industria de la tortilla. 


\section{Referencias}

1. J. P. N. Rosazza, Z. Huang, L. Dostal, T. Volm, B. Rousseau, Review: Biocatalytic transformations of ferulic acid: an abundant aromatic natural product. J. Ind. Microbiol. Biotechnol. 1995:15, 457-471.

2. S. Ou, K.C. Kwok, Review Ferulic acid: pharmaceutical functions, preparation and applications in foods. J. Sci. Food Agric. 2004: 84, 1261-1269.

3. N. E. Gámez de León, M. Sánchez-González Extracción químicoenzimática del AF presente en diferentes variedades de maíz mexicano. Rev. Salud Púb. Nutric. 2006: 11, 28.

4. Valladares-Juárez A. G., Recuperación de ácido ferúlico de subproductos y desechos de la industria de la tortilla. Tesis de Maestría en Ciencias con Orientación en Procesos Sustentables, UANL. 2007

5. G. Crini, P. M. Badot, Aplication of chitosan, a natural aminopolysaccharide, for dye removal from aqueous solutions by adsorption processes using batch studies: A review of recent literature. Progress in Polymer Science. 2008: 33, 399-447. 